\title{
Procesos cognitivos y metacognitivos en la solución de problemas de movimiento de figuras en el plano a través de ambientes computacionales
}

\author{
Nilson Genaro Valencia Vallejo* \\ Luis Bayardo Sanabria Rodríguez
}

Jaime Ibáñez Ibáñez

Artículo recibido: 17-12-2010 y aprobado: 04-05-2012

Resumen: La comprensión de conceptos sobre movimiento de figuras geométricas en el plano en ambientes computacionales dinámicos es un tema abordado en el aprendizaje de la geometría. La interacción con estos ambientes ayuda a los estudiantes a desarrollar competencias cognitivas y metacognitivas cuando se enfrentan a situaciones problemáticas. A partir de este proceso, la investigación desarrollada estudió la comprensión de conceptos en un contexto de solución de problemas de geometría que van de lo simple a lo complejo o viceversa. Como complemento de este proceso se describieron las estrategias cognitivas y en algunos casos metacognitivas que sigue el estudiante en su proceso de aprendizaje. Los resultados de esta investigación muestran que la interacción de los estudiantes en la solución de problemas gráficos que van de lo simple a lo complejo o viceversa, no muestran diferencias significativas. En relación a la comprensión de conceptos, se pudo establecer que el nivel de aprendizaje obtenido por los estudiantes cuando interactuaron con el ambiente computacional con apoyo de guías fue mejor. Al parecer las ayudas y la orientación del docente son un poderoso motivador en el aprendizaje, como queda demostrado en otros estudios.

Palabras clave: Comprensión de conceptos, ambientes de aprendizaje computacionales, geometría dinámica, cognición, metacognición, representación de conocimiento, cambio conceptual.

\section{Cognitive and metacognitive processes in problem-solving movement at the level of figures through computational environments}

Abstract: The understanding of concepts on geometric figures movement in the plane through computational environments is a topic addressed to geometry learning. Interaction with these environments helps students to develop cognitive and meta cognitive skills when facing problematic situations. This research project studied the understanding of concepts in the context of solving geometry problems from the simple to the complex or vice versa. As a complement to this process, the researchers identified the cognitive and meta cognitive strategies used by the students in their learning process. The results of this research show that students'interaction in solving graphic problems from simple to complex or vice versa, do not show significant differences. In relation to the understanding of concepts it was established that the level of learning achieved by students was higher when they interacted with the computational environment supported by guides. Apparently the teacher's guidance is a powerful motivator for students to learn.

Keywords: Understanding concepts, learning computational environments, geometry, dynamics, cognition, metacognition, knowledge representation, conceptual change.

\footnotetext{
* Universidad Pedagógica Nacional, Departamento de Tecnología. nvalencia@pedagogica.edu.co

* Universidad Pedagógica Nacional, Departamento de Tecnología. lubsan@pedagogica.edu.co

*** Universidad Pedagógica Nacional, Departamento de Tecnología. jibanez@pedagogica.edu.co
} 


\section{Introducción}

El contexto de la investigación que da origen a este documento se relaciona con la comprensión de conceptos, a partir de la interacción de los sujetos que solucionan problemas de movimiento de figuras en el plano, apoyados en escenarios computacionales. El estudio plantea un análisis cuantitativo sobre la comprensión de conceptos de dos grupos de estudiantes de educación media básica, que interactúan resolviendo problemas propuestos en un ambiente computacional, y un análisis cualitativo para explorar los procesos de razonamiento en el contexto de la solución de problemas de movimiento de figuras en el plano.

Este trabajo se enmarca en el desarrollo de investigaciones relacionadas con el estudio de procesos de razonamiento en ambientes gráficos, que vinculan a los aprendices en una interacción con un escenario de solución de problemas con el objeto de adquirir conocimiento. Un aspecto que surge de esta investigación es el efecto de utilizar ayudas combinadas en el aprendizaje de conceptos, tanto a nivel textual como a nivel gráfico. Se supone que la inclusión de estos elementos en un escenario de aprendizaje eleva el nivel de motivación para alcanzar logros en el aprendizaje.

De acuerdo a Simon (1996), el esfuerzo de solucionar un problema debe ser precedido del esfuerzo para comprenderlo. Según este autor, los sistemas para comprender son sistemas de aprendizaje. La identificación de constructos en la solución de un problema llevaría a percibir estructuras de símbolos manejados en el problema, diferencias en las estructuras representadas, operadores que alteran las estructuras y objetivos representados en modelos gráficos. Según Simon (1996), para poder comprender un problema cuando ha sido presentado a partir de estas entidades, se requiere una serie de acciones tales como: detectar diferencias, encontrar operadores relevantes, aplicar operadores y evaluar el progreso a través de la solución. El trabajo que podría evidenciar una persona cuando se enfrenta a resolver un problema de geometría dinámica incluye estos procesos, en razón a que los movimientos de las figuras se convierten en operadores que van configurando la imagen puesta como objetivo. La evaluación se va dando a medida que el individuo logra detectar los cambios de la forma geométrica que desea alcanzar. Se pensaría que el progreso alcanzado en la solución de este tipo de problemas, le sirva para poder comprender los conceptos que rodean el proceso de solución. En esta lógica, el individuo podría extraer conceptos básicos con los cuales resolvería problemas más generales. La conceptualización, en este caso, consiste en descubrir los objetos que son relevantes, sus propiedades y relaciones con los cuales se puedan describir los estados y movimientos para alcanzar un objetivo final.

Un aspecto importante de la interacción en estos escenarios son los procesos espaciales que se evidencian en la construcción de los modelos gráficos. Al respecto, Suwa y Motoda (1995) plantean dos clases de información para manejar este tipo de razonamiento: información visual e información conceptual. La información visual se obtiene del diagrama o la representación gráfica; esta incluye 
la configuración espacial, la forma del modelo gráfico y sus componentes. Según Ohlsson (1994), la información conceptual proviene del conocimiento previo del sujeto e incluye el conocimiento predictivo, utilizado para hacer inferencias acerca de la dinámica del modelo gráfico. Para estas situaciones de razonamiento, los diagramas o representaciones gráficas bidimensionales sirven como representaciones compactas de información espacial. En esta dimensión, los diagramas facilitan el posicionamiento de conocimiento relevante para solucionar el problema. Asimismo, los diagramas soportan las visualizaciones mentales de la disposición de los componentes del modelo sobre el cual se desea razonar. De acuerdo a Narayanan, Suwa y Motoda (1995), tales visualizaciones guían el razonamiento humano en la dirección de causalidad. Supongamos a un individuo que maneja conocimientos básicos acerca de figuras geométricas y se enfrenta a solucionar un problema de configuración geométrica como el mostrado en el Tangram; esta persona requiere razonar acerca de los procesos espaciales que se evidencian en la construcción del modelo gráfico.

\section{Revisión conceptual}

\section{Ambientes dinámicos para la comprensión de conceptos geométricos}

En la concepción de ambientes de aprendizaje para la geometría, Glass y Deckert (2001) distinguen dos escenarios: ambientes interactivos y ambientes de construcción estática. Los ambientes interactivos permiten al usuario modificar los objetos que presenta el entorno, manipulándolos a partir del movimiento de sus vértices y ejes hasta lograr la transición deseada. Los ambientes de construcción estática típicamente permiten a los estudiantes construir objetos geométricos en el computador, aplicando construcciones euclidianas comunes como medianas, vértices, lados, entre otros. Los ambientes estáticos permiten recordar una secuencia de construcciones para luego ser aplicadas en la construcción de un nuevo objeto. Sin embargo, los mismos ambientes no permiten manipular los objetos originales directamente y observar los efectos de esta manipulación de manera inmediata en la pantalla del computador.

Según Jones (2002), un escenario de geometría dinámica es un ambiente interactivo que provee un rango de herramientas para construir objetos geométricos, con una variedad de objetos primitivos como puntos, segmentos, líneas, entre otros. Este ambiente dispone de herramientas para la construcción de objetos como medianas, perpendiculares, paralelas, y herramientas para realizar transformaciones de objetos como la reflexión, la rotación y la traslación. Una vez se han dibujado los objetos se pueden tomar mediciones de ángulo, longitud, área, entre otros. La dinámica de estos ambientes proviene de la habilidad para dibujar o construir objetos definidos con la idea de configurar formas geométricas. Un ejemplo de este tipo de escenarios son el ambiente Cabri y el ambiente Logo. En la dinámica de este trabajo se plantea un escenario computacional que se asimila a un ambiente de aprendizaje dinámico con un solo objetivo, que es el movimiento de figuras en el plano; para ello se ha utilizado el modelo del Tangram como base para la constitución de objetos geomé- 
tricos generados por el movimiento de figuras planas.

Para alcanzar un nivel de comprensión, se requiere una serie de procesos que se enmarcan en lo que Chi y Ohlsson (2005) denominan aprendizaje complejo. Según estos autores, para el aprendizaje de conceptos se requieren los siguientes procesos: integración de información de varias fuentes, generación de inferencias, conectividad entre la información nueva y el conocimiento existente, recuperación apropiada de analogías, producción de explicaciones, coordinación de diferentes representaciones y perspectivas y abandono de conceptos previos de poca utilidad.

Con la finalidad de construir un ambiente dinámico para desarrollar estas habilidades, se prevé el nivel de comprensión que adquiere un individuo a partir del número de conceptos que logra asimilar. En este aspecto, Jones (2002) investiga la relación que existe entre un ambiente de geometría dinámica (Cabri) y la clase de pensamiento en la comprensión de conceptos geométricos que puede desarrollarse como resultado de las interacciones con el software. Su trabajo empírico examina cómo el uso de Cabri sirve como mediador en el aprendizaje de conceptos geométricos. Las conclusiones de este trabajo se enmarcan en los siguientes aspectos: los jóvenes que interactúan en el computador se fijan más en los resultados que muestra la pantalla, sin reflexionar sobre el proceso de construcción de la tarea; la manipulación de figuras en la pantalla no necesariamente conlleva a apreciar las propiedades conceptuales de las figuras geométricas.
En otro trabajo relacionado con el interés de la investigación desarrollada, Olkun (2003) compara el efecto del computador contra las manipulaciones concretas para el aprendizaje de la geometría bidimensional. El propósito del estudio consistió en comparar los efectos de las versiones de Tangram en computador y de manera concreta en el aprendizaje de un mismo contenido matemático, específicamente el razonamiento de formas geométricas bidimensionales y sus variantes. Esta investigación concluyó que solucionar problemas geométricos con manipulaciones, tanto en el computador como de manera concreta, tiene un efecto positivo en el razonamiento geométrico de los estudiantes. Los aprendices que interactuaron de manera concreta obtuvieron mejores resultados en el juego que los aprendices que interactuaron con el computador. Según el autor, este resultado se explica por la experiencia de los aprendices en la realización de manipulaciones concretas.

\section{Razonamiento a partir de la solución de problemas gráficos}

El esfuerzo de solucionar un problema debe ser precedido por el esfuerzo para comprenderlo; los sistemas para comprender son sistemas de aprendizaje. La identificación de constructos en la solución de un problema llevaría a percibir: estructuras de símbolos manejados en el problema, diferencias en las estructuras representadas, operadores que alteran las estructuras y objetivos representados en modelos gráficos. Según Simón (1996), para poder comprender un problema cuando ha sido presentado a partir de los elementos de la tarea mencionados, se requiere una 
serie de acciones tales como: detectar diferencias, encontrar operadores relevantes, aplicar operadores y evaluar el progreso a través de la solución. El trabajo que podría evidenciar una persona cuando se enfrenta a resolver un problema de geometría dinámica incluye estos procesos, en razón a que los movimientos de las figuras se convierten en operadores que van configurando la imagen puesta como objetivo. La evaluación se va dando a medida que el individuo logra detectar los cambios de la forma geométrica que desea alcanzar. Se pensaría que el progreso alcanzado en la solución de este tipo de problemas, le permita comprender los conceptos que rodean el proceso de solución; en esta lógica, el individuo estaría en capacidad de extraer conceptos básicos con los cuales podría resolver problemas más generales. La conceptualización, en este caso, consiste en descubrir los objetos que son relevantes, sus propiedades y relaciones con los cuales se puedan describir los estados y movimientos para alcanzar la meta.

Supongamos a un individuo que maneja conocimientos básicos acerca de figuras geométricas y se enfrenta a solucionar un problema de configuración geométrica como el mostrado en el Tangram; esta persona requiere razonar acerca de los procesos espaciales que se evidencian en la construcción del modelo gráfico. Según Narayanan et. al. (1995), se requieren dos clases de información para manejar este tipo de razonamiento: información visual e información conceptual. La información visual se obtiene del diagrama o la representación gráfica; esta incluye la configuración espacial, la forma del modelo gráfico y sus componentes. La información conceptual proviene del conocimiento previo del sujeto e incluye el conocimiento predictivo, utilizado para hacer inferencias acerca de la dinámica del modelo gráfico. Para estas situaciones de razonamiento, los diagramas o representaciones gráficas bidimensionales sirven como representaciones unificadas de información espacial, de tal forma que facilitan el posicionamiento de conocimiento relevante para solucionar el problema y soportan la representación mental de la disposición de los componentes del modelo sobre el cual se desea razonar.

Narayanan et. al. (1995), plantean una serie de pasos que son requeridos para resolver problemas del tipo de representaciones bidimensionales. Estos pasos los clasifican en siete tipos:

1. Observación del modelo gráfico o diagrama. Esta tarea consiste en observar el modelo o la disposición de la representación bidimensional para localizar y recuperar información espacial.

2. Recuperación del conocimiento conceptual. Conocimiento conceptual recuperado de la memoria de largo plazo. Este proceso consiste en recuperar la información de las representaciones descriptivas.

3. Recuperación de conocimiento inferencial. Conocimiento procedimental recuperado de la memoria de largo plazo, aplicable en la solución del problema. Se evidencia en el apareamiento y recuperación de reglas. 
4. Inferencia conceptual. Este proceso consiste en hacer inferencias basadas únicamente en el conocimiento conceptual, desde la memoria de corto plazo a la memoria de largo plazo.

5. Inferencia visual. Consiste en hacer inferencias basadas únicamente en la información visual del modelo gráfico.

6. Inferencia híbrida. Consiste en hacer inferencias basadas tanto en el conocimiento conceptual como en la información visual.

7. Visualización. Consiste en imaginar los comportamientos espaciales de los componentes en el modelo.

En síntesis, Narayanan et. al. (1995) definen el razonamiento diagramático como el acto de razonar y solucionar problemas, utilizando diagramas o gráficos como representaciones externas. Aunque los modelos gráficos o diagramas son representaciones estáticas externas, el razonamiento con estos modelos incluye simulaciones mentales de comportamientos que cambian las configuraciones descritas en ellos, lo cual puede ser soportado por procesos cognitivos fundamentales que son imaginados por naturaleza.

\section{Procesamiento de información a partir de imágenes dinámicas}

La animación o movimiento de figuras puede proporcionar a los aprendices información dinámica explicita, que de algún modo se considera implícita o inutilizable en gráficos estáticos. Sin embargo, cuando se incluyen cambios temporales en una escena visual, se introduce una demanda adicional, cuali- tativamente diferente de procesamiento de información. Para que estos cambios sean exitosos en los modelos mentales de los aprendices, ellos deben extraer información relevante del movimiento o animación de las figuras para ser incorporadas en su estructura de conocimiento. Las descripciones dinámicas ayudan a los sujetos a predecir patrones futuros de conocimiento, lo cual les permite hacer generalizaciones en la solución de otros problemas.

Una característica instruccional que distingue las gráficas dinámicas de las gráficas estáticas es su capacidad de describir directamente un cambio temporal. Según Lowe (1999), una consideración de las implicaciones perceptual y cognitiva de esta descripción sugiere que con algunas animaciones, los aprendices pueden enfrentar altos niveles de carga cognitiva que podrían ser regulados con alternativas estáticas.

De acuerdo a Lowe (2003), las animaciones o imágenes dinámicas típicamente incorporan tres tipos principales de cambios en las entidades gráficas que las componen:

- Transformaciones. Cambios de forma que involucran alteraciones a entidades gráficas con respecto a propiedades tales como: tamaño, forma, color y textura.

- Traslaciones. Cambios de posición que involucran el movimiento de entidades gráficas completas de un lugar a otro y pueden ser percibidas con respecto a los límites de la animación u otro elemento incluido en el despliegue de la animación. 
- Transiciones. Cambios de inclusión que involucran la aparición o desaparición de entidades gráficas de manera total o parcial. Este cambio puede ocurrir de varias formas: en el borde y fuera de los bordes de las gráficas o en entidades gráficas que son adicionadas o removidas en el plano de una pantalla de computador.

A pesar de que las investigaciones en aprendizaje con animaciones o imágenes dinámicas son superiores a los gráficos estáticos, como lo han demostrado Narayanan y Hegarty (2000), también existen evidencias de que la acumulación de efectos animados no siempre pueden ser beneficiosos. Según Schnotz, Böckheler y Grzondziel (1999), existen posibles razones para esta falta de beneficios que incluyen la imposición de un procesamiento de información excesiva en los aprendices y una reducción en el compromiso de los aprendices a procesar actividades relevantes en su aprendizaje. En esta dimensión, las animaciones que presentan imágenes dinámicas muy especializadas con un alto grado de complejidad visual podrían tener consecuencias negativas en la descripción de un dominio para los aprendices novatos, debido a la excesiva demanda en su procesamiento.

\section{Enfoque metodológico}

El enfoque metodológico se basa en un diseño cuasi experimental que utiliza un método cuantitativo para determinar la recolección y análisis de los datos, complementado con un estudio cualitativo exploratorio a través del análisis de protocolos. A partir del análisis estadístico se logra la integración de los resultados y la generación de las inferencias. El objetivo del estudio cualitativo es analizar los procesos de solución de problemas, para identificar las estrategias cognitivas de los estudiantes que intervienen en el juego del ambiente computacional. Este análisis se realiza a partir de la videograbación, donde se registra de manera fiel la información de dos sujetos que resuelven los mismos problemas realizados en el análisis estadístico.

\section{Población}

La población que participó en esta experiencia son estudiantes de grado séptimo de educación básica de una institución educativa distrital, con conocimientos básicos en geometría euclidiana, divididos en dos grupos. El entrenamiento de un primer grupo consistía en resolver problemas gráficos que iban de lo simple a lo complejo, mientras que el otro resolvía problemas de lo complejo a lo simple. Para el análisis de protocolos se seleccionaron seis estudiantes, que fueron instruidos para que verbalizaran la información que ellos atienden, mientras solucionan un problema. Este proceso se llevó a cabo con el ambiente computacional Misioneros y caníbales, con el propósito de no viciar el proceso investigativo e incluye la descripción verbal, explícita en "informes verbales concurrentes", donde el individuo habla en voz alta a medida que realiza alguna operación en el proceso de solución. La finalidad del entrenamiento a los estudiantes consiste en garantizar sus habilidades de verbalización en la etapa de interacción con el ambiente computacional. Una vez que los individuos interactúan en el ambien- 
te computacional, el proceso se registra a través de una videograbación para una posterior codificación y modelación de las estrategias de solución de problemas utilizadas por cada estudiante.

En función de complementar el protocolo, los estudiantes son entrevistados para que revelen el proceso que siguieron en la solución del problema. El objetivo del proceso retrospectivo es obtener información adicional que provea al estudio una comprensión rica y profunda de la situación de aprendizaje planteada. Los reportes verbales retrospectivos son verbalizaciones directas de los procesos cognitivos, donde la información se guarda en la memoria mientras se completa la tarea. Mucha información de la memoria de largo plazo puede ser reportada directamente o utilizada como señales de recuperación posteriormente (Ericsson y Simon, 1993). Concluido el anterior proceso, se analizan los registros verbales desarrollado por los estudiantes, a través del estudio y sistematización textual y gráfica de la videograbación.

\section{Ambiente de aprendizaje computacional}

El ambiente de aprendizaje computacional se elaboró en el lenguaje de programación orientado a objetos OpenScript de Toolbook Instructor 2004. El ambiente incluye dos entradas para el código de acceso y el nombre que identifican al usuario. La figura 1 presenta el ambiente computacional dividido en dos áreas: un área de herramientas y otra de trabajo.

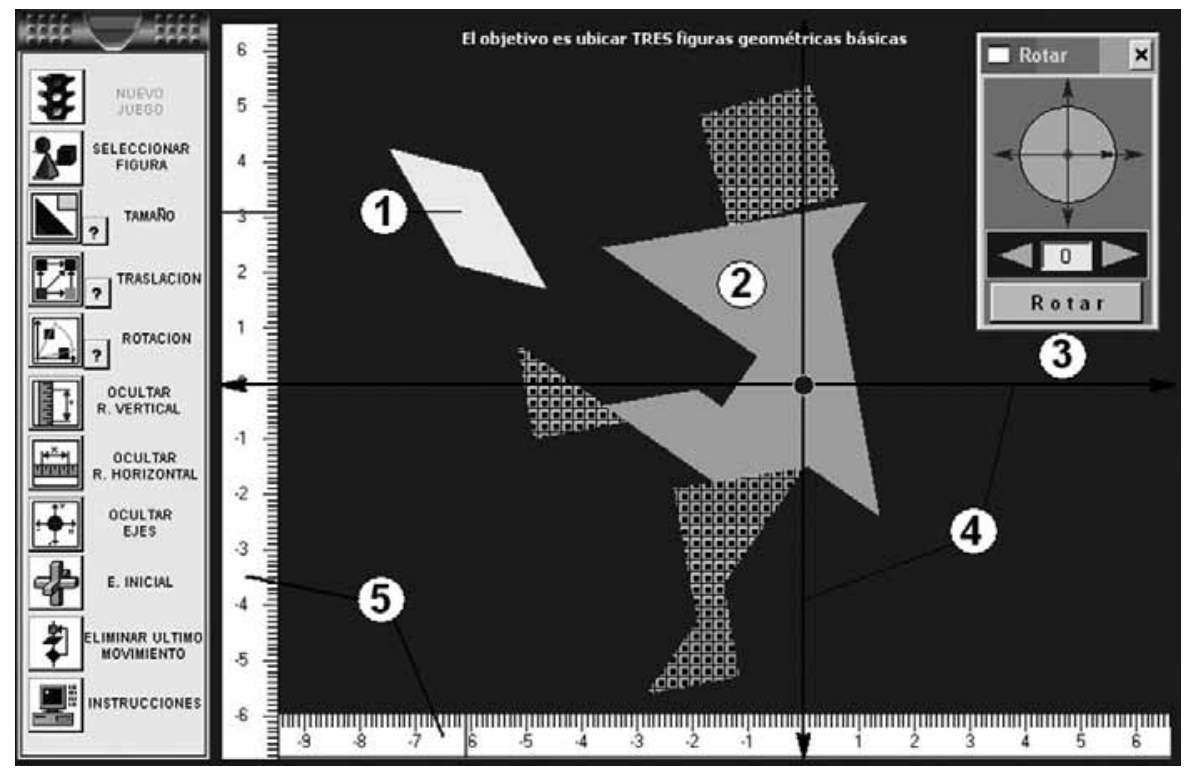

Figura 1. Ambiente computacional compuesto por: 1). Figura deslizante 2). Problema gráfico 3). Paleta de rotación de figuras 4). Eje cartesiano y 5). Escala métrica 
La zona de trabajo se diseñó con el propósito de posibilitar al estudiante el reconocimiento y manejo adecuado de las herramientas del ambiente y a su vez la interacción con la guía textual impresa.

El problema planteado a los sujetos consiste en colocar las figuras del Tangram que faltan, para completar una silueta que representa diferentes formas (ver figura 2). El nivel de dificul- tad depende de la cantidad de figuras que oculte el software: una, dos o tres figuras. El sujeto debe completarlas, identificándolas y ubicándolas en el espacio correspondiente para elaborar la composición final. Para realizar esta acción, el estudiante tiene la posibilidad de rotar la figura (rotación), ampliar o reducir el tamaño de la figura (homotecia) o trasladar la figura (traslación).

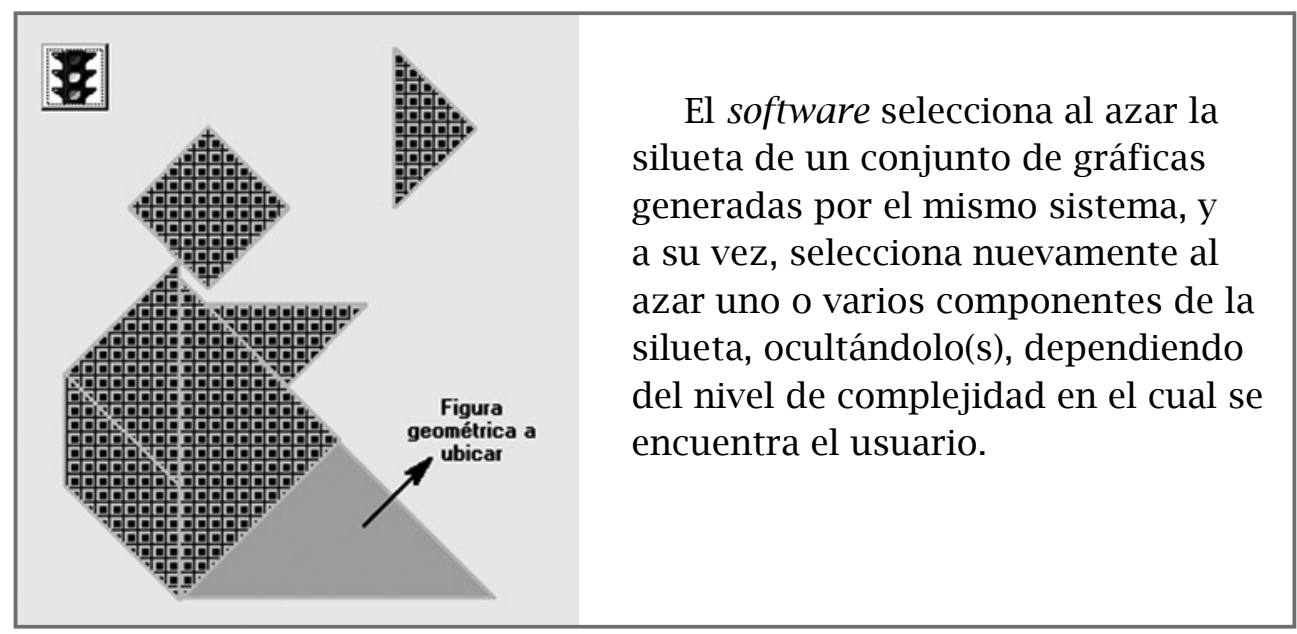

Figura 2. Modelo de problema

\section{Análisis de resultados}

\section{Análisis cuantitativo}

Los datos obtenidos fueron procesados usando el programa de computador Statistics de StatSoft Inc, versión 5.5. Para este análisis, los puntajes alcanzados por los estudiantes de cada grupo experimental se compararon mediante prueba $\mathrm{T}$. 


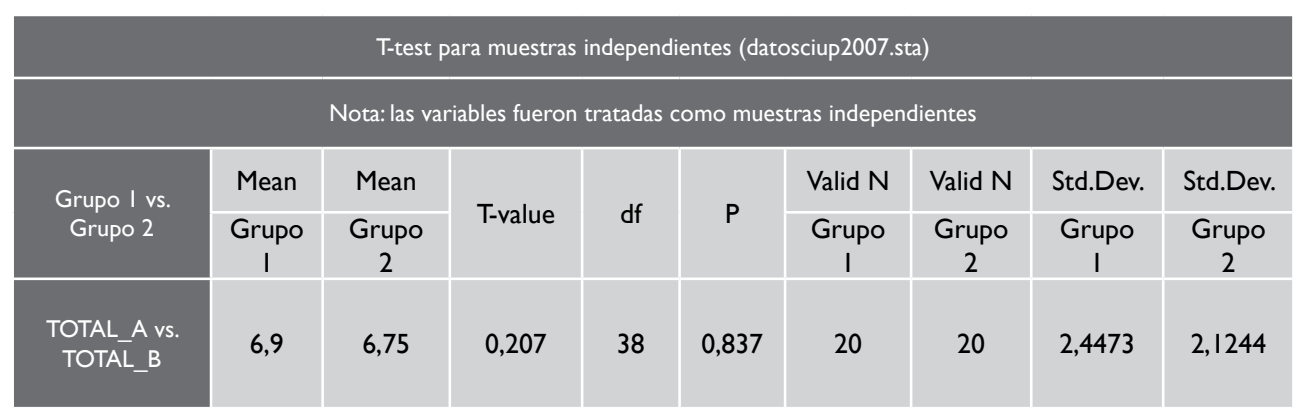

Tabla 1. Valores de la prueba T al comparar los puntajes totales obtenidos en la evaluación de los dos grupos

La tabla 1 presenta los valores de la prueba $\mathrm{T}$, al comparar los dos grupos en los puntajes totales de la evaluación. Estos puntajes corresponden a la suma de los puntajes de los tres movimientos (traslación, homotecia y rotación). En esta tabla se observan los valores de las medias (Mean) de los dos grupos, el valor de $\mathrm{T}$ (T-value), los grados de libertad (df), la probabilidad de error (p), casos válidos (Valid N) para los dos grupos y las desviaciones estándar (Std.Dev) de cada grupo.
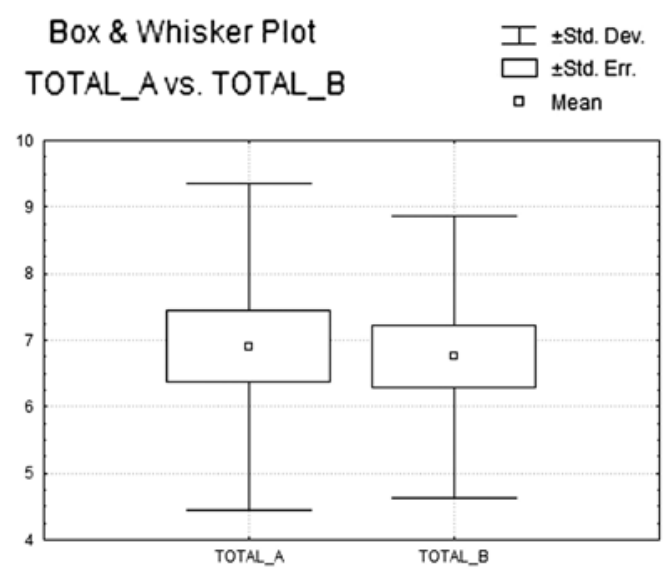

Figura 3. Comparación de medias y desviaciones estándar para los puntajes de la evaluación de los dos grupos
Se observa que el valor de $\mathrm{P}$ fue superior a 0.05 , lo que muestra que no existen diferencias significativas entre los puntajes totales de quienes solucionaron los problemas de menor a mayor nivel de complejidad (Grupo 1) y viceversa (Grupo 2). Ver figura 3.

La tabla 2 muestra los valores de la prueba T, al comparar los puntajes de la evaluación obtenida por los dos grupos que realizaron acciones de traslación, homotecia y rotación de manera independiente. En esta tabla se observan los valores de las medias (Mean) de los dos grupos, el valor de $\mathrm{T}$ (T-value), los grados de libertad (df), la probabilidad de error (P), casos válidos (Valid N) para los dos grupos y las desviaciones estándar (Std. Dev) de cada grupo. Se observa que en ningún caso el valor de $\mathrm{P}$ fue inferior a 0.05 , lo que indica que no existen diferencias significativas para los valores parciales de la evaluación, entre quienes solucionaron los problemas de menor a mayor nivel de complejidad (Grupo 1) y los estudiantes que solucionaron el juego de lo complejo a lo simple (Grupo 2). Lo anterior concuerda con las investigaciones realizadas por Olkun (2003), en relación al efecto del computador contra las manipulaciones concretas para el aprendizaje de la geometría bidimensional. 
T-test para muestras independientes (datosciup2007.sta)

\begin{tabular}{|c|c|c|c|c|c|c|c|c|c|}
\hline \multicolumn{10}{|c|}{ T-test para muestras independientes (datosciup2007.sta) } \\
\hline \multicolumn{10}{|c|}{ Nota: las variables fueron tratadas como muestras independientes } \\
\hline \multirow{2}{*}{$\begin{array}{l}\text { Grupo I } \\
\text { vs. } \\
\text { Grupo } 2\end{array}$} & Mean & Mean & \multirow[b]{2}{*}{ T-value } & \multirow[b]{2}{*}{ df } & \multirow[b]{2}{*}{$P$} & Valid N & Valid N & Std.Dev. & $\begin{array}{l}\text { Std. } \\
\text { Dev. }\end{array}$ \\
\hline & Grupo & $\begin{array}{c}\text { Grupo } \\
2\end{array}$ & & & & $\begin{array}{c}\text { Grupo } \\
\text { I }\end{array}$ & $\begin{array}{c}\text { Grupo } \\
2\end{array}$ & $\begin{array}{c}\text { Grupo } \\
\text { I }\end{array}$ & $\begin{array}{c}\text { Grupo } \\
2\end{array}$ \\
\hline $\begin{array}{l}\text { Traslación_I vs. } \\
\text { Traslación_2 }\end{array}$ & 2,5 & 2,8 & $-0,94$ & 38 & 0,35 & 20 & 20 & 1,1002 & 0,8944 \\
\hline $\begin{array}{c}\text { Homotecia_I } \\
\text { vs. } \\
\text { Homotecia_2 }\end{array}$ & 2,2 & 2 & 0,72 & 38 & 0,475 & 20 & 20 & 0,9515 & 0,7947 \\
\hline $\begin{array}{l}\text { Rotación_I vs. } \\
\text { Rotación_2 }\end{array}$ & 2,2 & 1,95 & 0,65 & 38 & 0,519 & 20 & 20 & I,28|4 & $\mathrm{I}, \mathrm{I} 459$ \\
\hline
\end{tabular}

Tabla 2. Valores de la prueba T al comparar los puntajes correspondientes a traslación, homotecia y rotación, obtenidos de manera independiente en los dos grupos

\section{Análisis cualitativo}

Este análisis se implementa para construir los modelos de procesamiento de información generados a partir de la solución de los problemas, sobre la base de protocolos de pensamiento en voz alta. El método de análisis de protocolos verbales, desarrollado por Ericsson y Simon (1993) como un método descriptivo que introduce el pensamiento en voz alta en la solución de problemas, es una técnica que utiliza este estudio para capturar el proceso de solución de un problema, o la toma de decisiones por parte del estudiante cuando hace la tarea para completar la forma geométrica que le aparece en la pantalla del computador.

Los pasos descritos a continuación son tomados como referentes en el análisis. Su aplicación recoge la verbalización del sujeto para codificarla y modelarla. Con este proceso se pretenden determinar las estrategias seguidas durante la solución del problema gráfico.

\section{Codificación de la información}

La codificación del material grabado se da en varias etapas. La primera etapa consiste en la trascripción de los datos. Esta operación se realiza de manera fiel. El siguiente párrafo muestra una parte de información transcrita de la videograbación de uno de los sujetos que participaron en la experiencia:

\section{"Selecciono el primer nivel.}

Tengo que ubicar la figura geométrica aquí. Primero lo traslado para que no sobrepase los límites. Ahora estoy agrandándolo con la homotecia para que quede del tamaño de la figura que tengo que ubicar; pero tengo que trasladarlo un poco más para que quede en el lugar preciso, para que no sobrepase los límites cuando realice la homotecia, o sea maximizarlo.

Ahora tengo que dejarlo más pequeño porque está muy grande. Sí, tengo que agrandar un poco la 
figura para que quede en el lugar de la figura. Ahora lo voy a rotar. Ahora lo estoy rotando para que quede acomodado en la figura. Ahora lo voy a mover para que quede en el lugar. Listo".

En esta verbalización se evidencia el manejo de una serie de procesos, que generan elementos de análisis para ubicar una figura en la silueta del entorno de la tarea.

La segunda etapa de codificación de segmentos, incluye la determinación de unidades de análisis. El proceso seguido en la investigación muestra instancias derivadas de la verbalización del sujeto que se traducen en cortes para determinar proposiciones como unidades de análisis. El párrafo anterior se segmenta como sigue.

S1. Selecciono el primer nivel. Tengo que ubicar la figura geométrica aquí.

S2. Primero lo traslado para que no sobrepase los límites.

S3. Ahora estoy agrandándolo con la homotecia para que quede del tamaño de la figura que tengo que ubicar.

S4. Pero tengo que trasladarlo un poco más para que quede en el lugar preciso, para que no sobrepase los límites cuando realice la homotecia, o sea maximizarlo.

S5. Ahora tengo que dejarlo más pequeño porque está muy grande. Sí, tengo que agrandar un poco la figura para que quede en el lugar de la figura.
S6. Ahora lo voy a rotar. Ahora lo estoy rotando para que quede acomodado en la figura.

S7. Ahora lo voy a mover para que quede en el lugar. Listo.

Cada segmento constituye una unidad de análisis que puede clasificarse en objetivos, estructuras de control, acciones determinadas por operadores y procesos evaluativos. Al hacer el análisis de los anteriores segmentos, se puede determinar una nominación para cada proposición. Esta clasificación se daría de la siguiente forma:

S1: corresponde a un objetivo que se plantea el sujeto.

S2, S6 y S7: corresponden a acciones acompañadas de operadores cognitivos (trasladar, rotar).

S3, S4 y S5: son estructuras de control que le permiten al sujeto calcular el tamaño de la figura que desea situar.

S7: además de realizar una operación, el sujeto evalúa la posición para determinar que el objeto está bien ubicado.

\section{Análisis de la información}

El análisis de la información que ha sido codificada se determina a través de episodios. En esta investigación cada episodio se identificó como el proceso que siguió el estudiante para ubicar de manera correcta una pieza en la silueta de la forma geométrica tomada como objetivo del problema. Los episodios se simplifi- 
can utilizando lógica de predicados. El siguiente ejemplo muestra el proceso de reducción a partir de la segmentación, con lo cual se determinan las cláusulas de esta forma de representación. La representación del episodio determina el proceso seguido por un sujeto en la ubicación correcta de una pieza.

Segmentos correspondientes al episodio de la ubicación de una pieza por uno de los sujetos:

S1. Tengo que combinar 2 figuras. Voy a empezar ahora por el triángulo.

S2. Ahora lo estoy moviendo para que quede donde va el triángulo y después agrandarlo. Listo.

S3. Lo estoy moviendo un poco para después agrandarlo y que no me sobrepase los límites.

S4. No me esperaba que se moviera hacia abajo.

S5. Ahora lo estoy moviendo hacia arriba, porque cuando lo agrando se va para abajo.

S6. Entonces me toca moverlo un poco hacia arriba.

S7. Ahora lo encojo un poco. Listo.

S8. Ahora lo voy acomodar, lo acomodo un poco.

S9. Ahora lo voy a rotar.

S10. Voy a rotarlo un poco al triángulo para acomodarlo en el lugar.

S11. Entonces ya solo tengo que moverlo para empezar a hacer la segunda figura.
S12. Como que me va tocar agrandarlo un poco porque está muу реqueño. Listo (fin primer episodio).

Los siguientes predicados representan el episodio anterior:

objetivo (g) $\mathrm{P}$ combinar (figura, a, b); empezar (figura, a).

mover (triángulo, dirección(x)) p mover (a, horizontal), localizar (a, destino).

localizar (a, destino) Р agrandar (a, tamaño(z)), mover (a, vertical), mover (a, vertical; horizontal), reducir (a, tamaño(z)), girar (a, izquierda), mover (a, vertical; horizontal).

La representación de los predicados lleva a la construcción de un modelo de grafos dirigidos, con el objeto de identificar las estrategias de solución del problema. Esta representación es una abstracción del episodio que representa la secuencia de estados de solución y los operadores en cada transición de estado (Newell y Simon, 1972).

A continuación se muestran los grafos más representativos de los dos sujetos, con los cuales se hizo el análisis de las estrategias seguidas en el proceso. El grafo de la figura 4 corresponde al episodio anterior, codificado con lógica de predicados. En este grafo se representa la estrategia seguida por un estudiante cuando resuelve problemas que inician en un nivel de dificultad alto para finalizar en un nivel de dificultad bajo. 


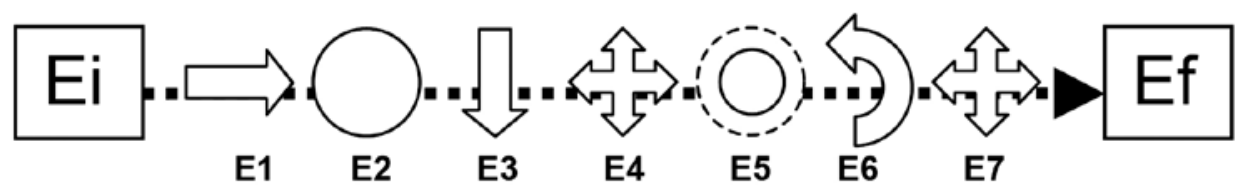

Figura 4. Grafo del tercer episodio -Problema 2- Segundo nivel. Sujeto 1

En la figura 5 se representan las convenciones implementadas para la elaboración de los grafos.

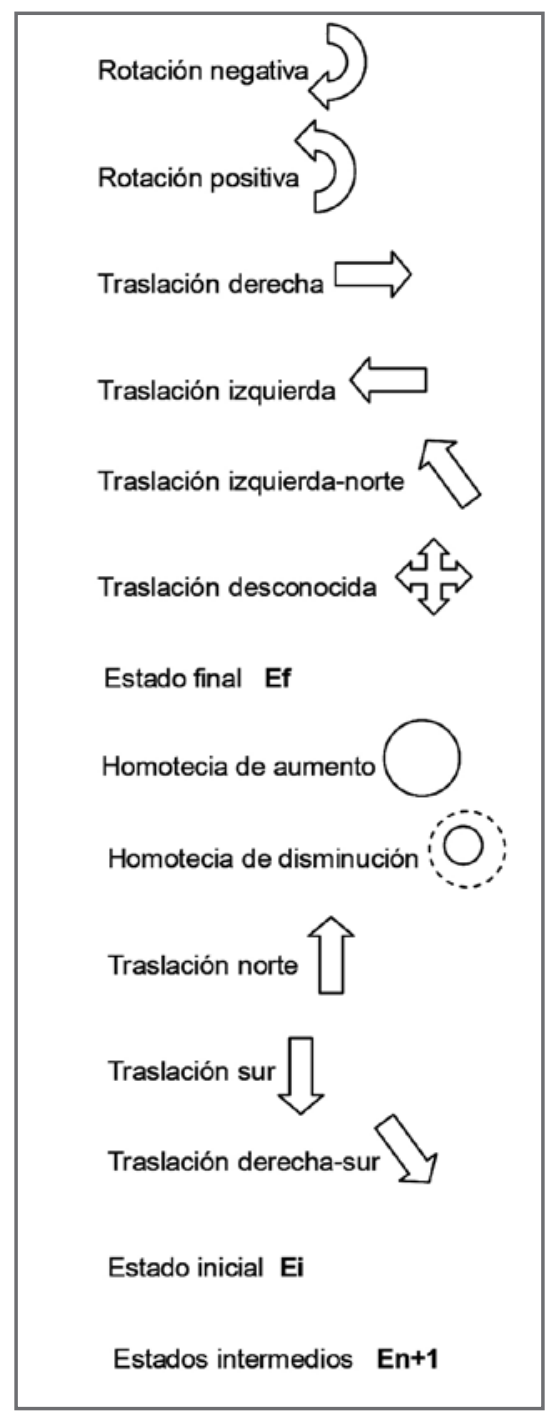

Figura 5. Convenciones utilizadas para la elaboración de los grafos
El grafo de la figura 4 muestra una estrategia centrada en la localización de la figura, donde se evidencian situaciones como inferir el número y la forma de figuras que encajan en la silueta; este tipo de razonamiento es un proceso metacognitivo, donde el aprendiz comprende el problema y cree acerca de los juicios en la asignación de recursos cognitivos, referidos a las operaciones que planea para resolver el problema. Otras situaciones que hacen parte de la estrategia tienen que ver con las aproximaciones ideadas por el aprendiz para lograr la meta, que se relacionan con la aplicación de operadores como mover la figura, agrandarla y probarla para localizar el objeto en su destino y el control que realiza sobre estas operaciones para precisar la ubicación de la figura en el lugar planeado. Estos procesos metacognitivos están validados en los planteamientos de Jacobs y Paris (1987), Flawell (1976) y Brown (1987). Con la aplicación de operadores se podría pensar que el sujeto, sin tener un conocimiento suficiente acerca de los conceptos de traslación, rotación y homotecia, utiliza operadores que evidencian la aplicación de estos conceptos. Este factor coincide con el planteamiento teórico de Chi (2007) en lo que se refiere al cambio conceptual, donde el conocimiento relacionado que tiene el sujeto lo obliga a adicionar nuevo conocimiento a su memoria, en algunos casos, o a comple- 
tarlo, en otros, a medida que avanza en su aprendizaje. En síntesis, podemos inferir, después de haber analizado el episodio, que el sujeto comprendió el problema por las acciones que llevó a cabo, tales como: detectar diferencias en la construcción geométrica, encontrar operadores relevantes como los utilizados, aplicarlos y evaluar el progreso a través de la localización del objetivo. Esto se ajusta al planteamiento de Simon (1996) con relación a las acciones realizadas en el proceso de comprensión de un problema.

Cuando el estudiante se enfrenta a resolver la secuencia de problemas de acuerdo al grado de dificultad, se observa la utilización de la misma estrategia, lo cual indica que existe una reafirmación de su conocimiento y una consolidación del nivel de precisión de la estrategia manejada. Se pensaría que este proceso de replicar la estrategia podría llevar al individuo a elaborar patrones mentales que le permiten hacer generalizaciones en la solución de otros problemas, como lo plantea Lowe (2003).

En el análisis del segundo sujeto se utilizó la misma lógica que con el primero. El grafo corresponde a la estrategia seguida por el sujeto 2 en un nivel de dificultad fácil, considerando que el crecimiento hacia lo más complejo opera con la solución de otros problemas. La figura 6 muestra la representación de un episodio correspondiente al primer ejercicio, que consistía en ubicar un objeto para completar una forma geométrica.

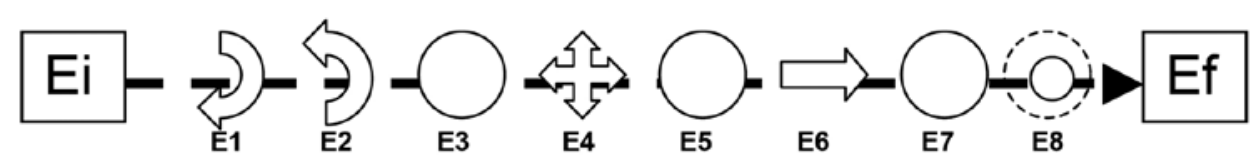

Figura 6. Grafo -Problema 3- Primer nivel. Sujeto 2

La estrategia utilizada por este sujeto cambia con respecto al anterior. El grafo muestra una estrategia centrada en la transformación de la figura, antes de localizarla (figura 6). En el análisis se observa cómo el sujeto 2, aplica operadores relacionados con el cambio de tamaño de la figura antes de ubicarla en su estado final. Esto lleva a pensar que el proceso metacognitivo seguido por el sujeto, se sitúa en hacer una planeación para llegar al estado final, relacionado con encajar la figura en la silueta. En este tipo de razonamiento, se infiere que el aprendiz comprende el problema y asigna recursos cognitivos aprendidos previamente para resolver el problema. Esto concuerda con una de las condiciones del cambio conceptual planteadas por Chi (2007), donde el sujeto tiene algunos conocimientos correctos previos que enriquece cuando encuentra la solución del problema. Las acciones seguidas en la estrategia se relacionan con la aplicación de operadores de transformación de la figura, como agrandarla, rotarla y probarla antes de localizarla en su destino, y el control que realiza sobre estas operaciones señala un manejo del nivel de precisión, lo cual indica que su proceso de razonamiento se basa en un nivel de comparación de 
los límites de la figura a construir y las operaciones cognitivas que realiza sobre el objeto que planea ubicar. Estos procesos metacognitivos, lo mismo que el sujeto anterior, se evidencian en la teoría de Jacobs y Paris (1987), Flawell (1976) y Brown (1987).

La aplicación de operadores del sujeto 2, hace suponer un manejo de conocimiento de los conceptos aplicados en la solución del problema, con lo cual se puede inferir que el sujeto maneja un nivel de comprensión del problema, planeando la aplicación de operadores precisos que lo llevan a establecer diferencias al comparar su trabajo con el estado final de solución. Con esto aplica una estrategia medio-fin que le permite acercarse a la solución en la medida que va evaluando sus acciones. Esto se asimila a la teoría de solución de problemas planteada por Simon (1996).

A medida que el sujeto avanza en la solución de los siguientes problemas de la secuencia, existe un incremento en la planeación de las acciones, lo cual indica que el número de operadores aumenta, mostrando el manejo de mayor variedad de conceptos que vienen a enriquecer el conocimiento del sujeto en el proceso de solución del problema. Este hecho queda demostrado en la teoría del cambio conceptual de Chi (2007).

Para hacer una síntesis de los resultados de las estrategias manejadas por los dos sujetos, se han definido los árboles de decisión que explican, de manera general, el comportamiento de los individuos durante el proceso de solución de los problemas gráficos planteados por el ambiente computacional. En la figura 7 se representa el comportamiento y las acciones de los sujetos para solucionar el problema. Los diagramas presentan la existencia de estrategias comunes utilizadas por los sujetos, donde existen semejanzas en la percepción del ambiente de la tarea y la representación del espacio del problema, y diferencias en la elección del camino de solución.

Las estrategias corresponden a acciones repetitivas en el proceso de solución del problema. Estas estrategias han sido plenamente identificadas en cada episodio. Su análisis coincide con el resultado de los grafos tratados anteriormente.

El sujeto 1 maneja una estrategia consistente en dos pasos: localización del objetivo y ajuste en el tamaño. Para ello ejecuta operaciones de traslación y aumento de tamaño o disminución y rotación. Su proceso de control consiste en comparar la posición. El sujeto 2 maneja una estrategia consistente en tres pasos: ajuste del tamaño, comparación con el estado final y localización del objetivo. Para su ejecución utiliza operadores de aumento y disminución de tamaño, rotación, traslación y ajuste en la posición. El sistema de control que utiliza es comparar el tamaño. Si observamos los dos procesos podemos encontrar acciones metacognitivas de planeación y control para lograr las metas propuestas, como lo proponen Jacobs y Paris (1987), Flawell (1976) y Brown (1987). Los dos modelos evidencian el manejo de conocimientos previos: mientras para el sujeto1 los conocimientos previos son escasos, lo cual lo lleva a ejecutar acciones imprecisas, el sujeto 2 tiene un mayor nivel conceptual, lo que le permite calcular con mayor precisión la planeación de sus acciones. Esta mirada se comprueba con la teoría del cambio conceptual manejada por Chi (2007). 


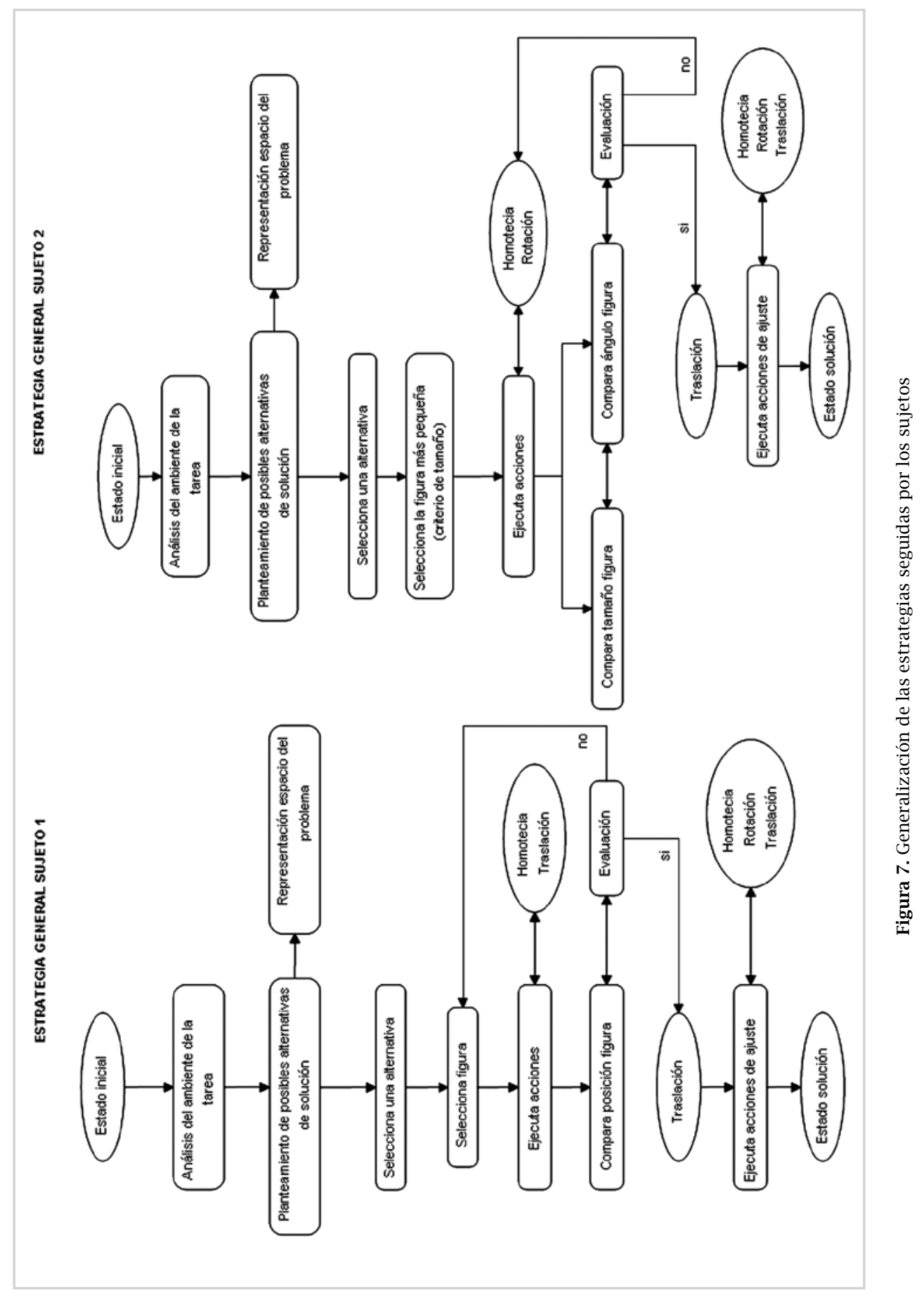




\section{Conclusiones}

La investigación se sustenta en el aprendizaje con apoyo de ambientes computacionales, en un contexto dominado por el estudio de conceptos, relaciones geométricas y métricas, a partir de la interacción con imágenes dinámicas para la comprensión de conceptos. El estudio pretende mostrar que la interacción con estos ambientes ayuda a los estudiantes a desarrollar competencias cognitivas y metacognitivas cuando se enfrentan a la solución de problemas. A partir de este proceso, la investigación planteada estudia la comprensión de conceptos en un contexto de solución de problemas de geometría, que van de lo simple a lo complejo o viceversa. Para el análisis metacognitivo se hace una descripción de las estrategias cognitivas y metacognitivas que sigue el estudiante en su proceso de aprendizaje. Con esta concepción la investigación responde a los siguientes interrogantes:

¿Existen diferencias significativas en la comprensión de conceptos sobre movimiento de figuras geométricas, entre un grupo de estudiantes que resuelve problemas que van de lo simple a lo complejo y otro que lo hace de lo complejo a lo simple?

¿Qué estrategias cognitivas se identifican en la interacción de un grupo de estudiantes que resuelve problemas de movimiento de figuras geométricas en el espacio, apoyado en escenarios computacionales?

Para dar respuesta a la primera pregunta y con base en los resultados estadísticos, se pudo observar que la interacción de los grupos en la solución de problemas gráficos que van de lo simple a lo complejo o viceversa, no muestra diferencias significativas. Esto confirma los resultados de la investigación realizada por Olkun (2003), donde concluye que a pesar de existir efectos positivos en el razonamiento geométrico de los estudiantes acerca de formas geométricas bidimensionales, no se mostraron diferencias significativas entre un grupo que resolvió problemas geométricos en un ambiente computacional y otro que lo hacía en un medio real.

Con relación a la comprensión de conceptos, se pudo establecer que el nivel de aprendizaje obtenido por los estudiantes, cuando interactuaron con el ambiente computacional con apoyo de guías, fue mejor, en razón a la ayuda que se les ofreció. Sus puntajes alcanzados muestran que la guía sí ayudo en el proceso. Lo cual se sustenta con la investigación de Jones (1998), donde se concluye que para comprender un concepto, no basta únicamente con el ambiente computacional, también se requiere la mediación del profesor u otro medio. La intervención del profesor a través de la guía utilizada, como se mostró en esta investigación, centró la atención del estudiante en la formación de los conceptos relacionados con traslación, rotación y homotecia, que al ser utilizados en la solución de los problemas más complejos, le permitió actuar con más eficiencia. La conclusión que surge de este análisis es que con la ayuda del profesor o con la inclusión de un apoyo en el proceso de aprendizaje, se equilibra el tiempo que utilizan los estudiantes cuando resuelven problemas que van de lo simple a lo complejo o viceversa. Esto queda demostrado una vez más en los resultados obtenidos por Jones (1998). 
En esta dimensión, se establece que la manipulación de figuras en la pantalla no necesariamente conlleva a apreciar las propiedades conceptuales de las figuras geométricas: para ello se requiere la mediación de un agente externo que oriente el proceso de aprendizaje.

En relación a la segunda pregunta, acerca de las estrategias obtenidas en el proceso de solución, se pudieron evidenciar dos tipos de estrategias: una, relacionada con localización del objetivo y ajuste en el tamaño de los objetos de acuerdo a los límites de la forma geométrica que se debía completar, y otra consistente en el ajuste del tamaño de los objetos, la comparación con el estado final y la localización del objetivo a partir de la meta propuesta. Estos componentes de cada una de las estrategias llevaron a los sujetos a aplicar conceptos de rotación, traslación y homotecia que fueron validados posteriormente en la prueba estadística. Al observar los dos procesos, podemos encontrar acciones metacognitivas de planeación y control para lograr la meta de completar una forma geométrica, lo cual queda demostrado en la teoría de Jacobs y Paris (1987), Flawell (1976) y Brown (1987). Una conclusión que se deduce al analizar el protocolo verbal del comportamiento de los sujetos, consiste en la activación de procesos metacognitivos ocasionada por el manejo de figuras dinámicas. Esta activación se ve claramente reflejada en la planeación y control de las acciones, que obligan a los sujetos a razonar acerca de la manera de resolver los problemas. Lo anterior se complementa con los resultados de la investigación de Olkun (2003) por un lado, donde se concluye que solucionar problemas geométricos con manipulaciones en el computador tiene un efecto positivo en el razonamiento geométrico de los estudiantes, y por otro, con la investigación de Glass y Deckert (2001), concluyendo que los estudiantes que utilizan ambientes computacionales para observar matemáticamente patrones precisos y formar conjeturas con base en estos patrones, pueden proveer experiencias ricas en razonamiento formal y mejorar la comprensión de conceptos geométricos.

El análisis de los protocolos permite inferir la elaboración de modelos mentales, a partir de la información visual obtenida de los modelos gráficos del ambiente computacional. Los procesos observados revelan la información visual a partir de la representación gráfica y conceptual derivada de los conceptos previos. Este hallazgo es congruente con los planteamientos de Narayanan et. al. (1995).

Otro hallazgo se refiere a las diferencias en el manejo de conocimientos previos. Este es un factor que se resalta en los modelos analizados, lo cual coincide con la teoría del cambio conceptual de Chi y Roscoe (2002), que lleva a suponer la existencia de variantes en el manejo de conceptos previos. Mientras algunos sujetos manejan conocimientos previos correctos, otros se basan en conocimientos relacionados.

Respecto del manejo de operadores cognitivos, inferidos del análisis del protocolo, se pudo concluir que el ejercicio de utilizar figuras dinámicas es una tarea que involucra tres tipos de acciones: la primera se relaciona con las transformaciones de las figuras, para 
ajustarlas al contexto de solución del problema que está representado en la configuración de una forma geométrica; la segunda, se refiere a las traslaciones de estas figuras con el fin de localizarlas con un mayor nivel de precisión; y la tercera a transiciones para determinar cambios de estado cuando se evalúan las figuras que no corresponden y se hace necesario utilizar unas nuevas. Lo anterior evidencia el manejo de las estrategias de los sujetos y es una confirmación del planteamiento de Lowe (2003), respecto a los tipos de cambios en las entidades gráficas.

Las posiciones anteriores indican que resolver problemas de geometría dinámica en escenarios computacionales, es una estrategia importante para la elaboración de modelos mentales, lo cual posibilita la comprensión y transferencia de aprendizaje de los estudiantes en la construcción de tareas relacionadas con el desarrollo de competencias cognitivas y metacognitivas. En síntesis, se infiere que la ayuda y la orientación del docente es un poderoso motivador en el aprendizaje, como queda demostrado en otros estudios.

Desde el punto de vista pedagógico, esta investigación abre una posibilidad de aprendizaje de la geometría con el uso de ambientes computacionales basados en el manejo de imágenes dinámicas. Su potencial a nivel cognitivo puede facilitar la progresión del aprendizaje en los diferentes niveles del modelo de Van Hiele (1986). Finalmente, se prevé que el uso de estos ambientes de aprendizaje ayuden en los procesos de cambio conceptual de los sujetos que tienen un manejo conceptual incorrecto, o requieren completar los vacíos existentes en su estructura cognitiva, en concordancia con el planteamiento teórico de Chi (2007).

\section{Referencias}

Brown, A.L. (1987). Metacognition, executive control, self-regulation, and other mysterious mechanisms. In F. Weinert \& R. Kluwe (Eds.), Metacognition, Motivation, and Understanding. New Jersey: Lawrence Erlbaum.

Chi, M.T.H. \& Ohlsson, S. (2005). Complex declarative learning. In K.J. Holyoak \& R.G. Morrison (Eds.), The Cambridge Handbook of Thinking and Reasoning (pp. 371-399). Cambridge University Press.

Chi, M.T.H. \& Roscoe, R.D. (2002). The processes and challenges of conceptual change. In M. Limon \& L. Mason (Eds), Reconsidering Conceptual Change: Issues in Theory and Practice (pp. 3-27). Netherlands: Kluwer Academic Publishers.

Chi, M.T.H. (2007). Three types of conceptual change: Belief revision, mental model transformation, and categorical shift. To appear in S. Vosniadou (Ed.), Handbook of research on conceptual change. Hillsdale, NJ: Erlbaum.

Ericsson K.A \& Simon H.A. (1993). Protocol Analysis: Verbal Reports as Data MIT Press. Cambridge, MA.

Flavell, J. H. (1976). Metacognitive aspects of problem solving. En L.B. Resnick (Ed.), The Nature of Intelligence. Hillsdale. NJ: Erlbaum.

Glass, B. \& Deckert, W. (2001). Making better use of computer tools in geometry. Mathematics Teacher, 94 (3), 224-228.

Jacobs, J.E. \& Paris, S.G. (1987). Children's metacognition about reading: Issues 
in definition, measurement and instruction. Educational Psychologist, 22, 255-278.

Jones K. (1998). The Mediation of Learning within a Dynamic Geometry Environment. In A. Olivier \& K. Newstead (Eds), Proceedings of the 22nd Conference of the International Group for the Psychology of Mathematics Education (pp. 96-103). Vol. 3. South Africa: University of Stellenbosch.

Jones, K. (2002). Implications for the classroom: Research on the use of Dynamic Software. Micromath Autumn, 18 (3), $18-20$.

Lowe, R.K. (1999). Extracting information from an animation during complex visual learning. European Journal of Psychology of Education, 14, 224-225.

Lowe, R.K. (2003). Animation and learning: selective processing of information in dynamic graphics. Learning and Instruction, 13, 157-176.

Narayanan, N.H. \& Hegarty, M. (2000). Communicating dynamic behaviors: are interactive multimedia presentations better than static mixed-mode presentations? En Diagrams 2000 conference. Scotland: University of Edinburgh.

Narayanan, N.H., Suwa, M. \& Motoda, H. (1995). Hypothesizing Behaviors from Device Diagrams. In J. Glasgow, N.H.
Narayanan y B. Chandrasekaran (Eds.), Diagrammatic Reasoning: Cognitive and Computational perspectives (pp. 501-534). Menlo Park, CA: AAAI Press/ The MIT Press.

Newell, A. \& Simon, H.A. (1972). Human problem solving. Englewood Cliffs. Nueva Jersey: Prentice-Hall.

Ohlsson, S. (1994). Declarative and procedural knowledge. En T. Husen \& T. Neville-Postlethwaite, (Eds.), The International Encyclopedia of Education (Vol. 3, 2nd ed., pp.1432-1434). London, UK: Pergamon Press.

Olkun, S. (2003). Comparing Computer versus Concrete Manipulatives. Learning 2D Geometry. JournaI of Computers in Mathematics and Science Teaching, 22(1), 43-56.

Schnotz, W., Böckheler, J. \& Grzondziel, H. (1999). Effects of visualization on the structure and applications of mental models. En XXVI International Congress of the Psychology. Montreal, Canada.

Simon, H.A. (1996). The Sciences of the Artificial. Library of Congress. Massachussetts, MIT: Massachussetts Institute of Technology.

Van Hiele, P.M. (1986). Structure and insight: A theory of mathematics education. Orlando, FL: Academic Press. 